\section{A) Check for updates}

Cite this: Polym. Chem., 2018, 9, 1684

Received 20th November 2017 Accepted 20th December 2017 DOI: 10.1039/c7py01945a rsc.li/polymers

\title{
3D-printing of dynamic self-healing cryogels with tuneable properties $\uparrow$
}

\author{
Milena Nadgorny, ${ }^{a}$ Joe Collins, ${ }^{a}$ Zeyun Xiao, ${ }^{a}$ Peter J. Scales ${ }^{a}$ and \\ Luke A. Connal (iD *b
}

\begin{abstract}
We report a novel synthetic and processing methodology for the preparation of doubly dynamic, selfhealing, 3D-printable macroporous gels. 3D-printable oxime hydrogels were prepared by cross-linking poly( $n$-hydroxyethyl acrylamide-co-methyl vinyl ketone) (PHEAA-co-PMVK) with a bifunctional hydroxylamine. 3D-printed oxime hydrogels were subjected to post-printing treatment by thermally induced phase separation (TIPS), which facilitated the formation of hydrogen bonding and oxime cross-links, and dramatically increased the mechanical strength of soft oxime objects in a well-controlled manner by up to $1900 \%$. The mechanical properties of the cryogels were tuned by freezing conditions, which affected the microstructure of the cryogels. These doubly dynamic 3D-printed cryogels are macroporous, exhibit outstanding swelling performances, and can fully, rapidly and autonomously self-heal.
\end{abstract}

\section{Introduction}

Self-healing materials possess a unique capability to recover from damage, thereby restoring their structure and function. Owing to their self-healing properties these nature-mimicking materials also derive the benefit of a prolonged lifetime and offer alternative solutions for damage repair. ${ }^{1,2}$ The development of self-healing materials has attracted a lot of attention, $^{2-5}$ however, very few studies focus on their processability. ${ }^{6,7}$

Three-dimensional printing (3DP) has emerged as a promising technology for advanced manufacturing of objects fabricated layer-by-layer from a digital model. ${ }^{8-10}$ Potential applications of 3DP have been successfully explored in a myriad fields, such as tissue engineering, ${ }^{11-14}$ catalysis, ${ }^{15}$ actuation, ${ }^{16}$ and sensing. ${ }^{17}$ To expand the utility of 3DP, development of new printable materials is highly desirable and is becoming a topic of significant scientific interest. ${ }^{10,15}$ A particularly intriguing group of materials is functional hydrogels. These materials can be exploited as actuators and shape morphing devices, ${ }^{6,16,18}$ act as biomimetic tissue engineering scaffolds, ${ }^{8,11,12,14}$ or exhibit self-healing capabilities. ${ }^{6}$ Retaining large volumes of solvent, these 3D polymer networks are usually prone to poor mechanical robustness. ${ }^{2,19}$ Moreover,

\footnotetext{
${ }^{a}$ Department of Chemical and Biomolecular Engineering, The University of Melbourne, Parkville 3010, VIC, Australia

${ }^{b}$ Research School of Chemistry, Australian National University, Canberra, Australian Capital Territory 2601, Australia. E-mail: luke.connal@anu.edu.au $\dagger$ Electronic supplementary information (ESI) available. See DOI: 10.1039/ c7py01945a
}

3DP by commonly used extrusion-based technologies imposes additional restrictions on the mechanical strength and structural integrity of 3D-printed gels, making this process extremely challenging. ${ }^{6}$

To be extrusion compliant, inks must be formulated within a well-specified rheological parameter range, ${ }^{6,9,12}$ making them even less versatile and softer than their non-printable analogues. To overcome these limitations, numerous techniques have been developed to reinforce soft 3D-printed objects. Well-known approaches include secondary (post-printing) cross-linking with ultraviolet (UV) light, ${ }^{8,11,12,14}$ chemical or physical cross-linking by covalent or ionic cross-linkers, ${ }^{6,8,19}$ evaporation induced curing, ${ }^{20}$ and thermally induced gelation. ${ }^{21,22}$ Despite their popularity, these methods rely on the addition of external compounds, such as photo-initiators or small molecules, ${ }^{8}$ or require a sophisticated design of polymers, which can exhibit concentration or temperature triggered self-assembly. ${ }^{20-22}$

Herein, we explore thermally induced phase separation (TIPS) as a post-printing reinforcement technique, which enables the fabrication of macroporous 3D-printed objects. Post-printing freeze-thaw cycles induce thermodynamically driven cross-linking of the polymer rich phase, resulting in well-controlled mechanical stabilization of soft gels. The macroporous structure of cryogels contributes to their improved mechanical strength. ${ }^{23}$ Recently, TIPS in combination with 3DP was reported as a useful approach to tailor the surface morphology of polymeric scaffolds. ${ }^{24}$ In this work, TIPS is explored as a novel tool for post-printing reinforcement of soft 3D-printable gels. More importantly, the mechanical properties of these self-healing cryogels are tuneable and can 
be modulated via the conditions of the freeze-thaw process. We design a doubly dynamic self-healing network by the synthesis of poly(n-hydroxyethyl acrylamide-co-methyl vinyl ketone) (PHEAA-co-PMVK). The ketone groups are harnessed to form oxime bonds with a bifunctional hydroxylamine crosslinker, and therefore enable the formation of stable dynamic covalent bonds, making the gel self-healable. PHEAA has been chosen owing to its excellent water solubility, eliminating the need for organic solvents. More importantly, the amide and the hydroxyl groups of HEAA units have a strong ability to form hydrogen bonds, which enable physical cross-linking during TIPS and make the self-healing cryogels doubly dynamic. First, oxime gels are formed. These soft self-healing gels can fully and autonomously recover from damage, ${ }^{3,25}$ and are 3D-printable. Post-printing TIPS treatment makes these gels doubly dynamic by facilitating the formation of hydrogen bonds, and improves the mechanical robustness of the soft objects in a well-controlled manner.

\section{Experimental section}

\section{Materials}

Chemicals were used as received unless otherwise stated. Triphenylphosphine (Alfa Aesar, 99\%), diisopropyl azodicarboxylate (DIAD, Sigma Aldrich, 98\%), n-hydroxyphthalimide (Alfa Aesar, 98\%), tetraethylene glycol (TEG, Alfa Aesar, 99\%), hydrochloric acid (37\%, RCI Labscan), potassium hydroxide (analaR NORAMPUR, 85.9\%), and hydrazine monohydrate (Sigma Aldrich, 98\%) were used. Tetrahydrofuran (THF, 99.8\%), anhydrous diethyl ether (99.5\%), dichloromethane (DCM, 99.8\%) and methanol (99.8\%) were purchased from Chem-Supply. Absolute ethanol (100\%, VWR) and Rhodamine B (Sigma Aldrich) were used. $N$-Hydroxyethyl acrylamide (HEAA, Sigma Aldrich, 97\%) and methyl vinyl ketone (MVK, Sigma Aldrich, 90\%) were purified by passing through a basic alumina column to remove the inhibitors. Azobisisobutyronitrile (AIBN, Sigma Aldrich, 98\%) was recrystallized from methanol. Dialysis tubing (CelluSep, regenerated cellulose membrane, T-series, $6000 \mathrm{MWCO}$ ) was submerged in deionized water for 20 minutes prior to use.

\section{Instrumentation}

Size exclusion chromatography (SEC) was performed on a Shimadzu Liquid Chromatography system equipped with a Shimadzu RID-10 refractometer $(\lambda=633 \mathrm{~nm})$ and a Wyatt 3-angle light scattering detector, with three Waters Ultrahydrogel columns in series ((i) $250 \AA$ porosity, $6 \mu \mathrm{m}$ diameter bead size; (ii) and (iii) linear, $10 \mu \mathrm{m}$ diameter bead size) for separation. Milli-Q water with 0.1 vol\% TFA was used as an eluent. Average molecular weights $\left(M_{\mathrm{n}}\right.$ and $\left.M_{\mathrm{w}}\right)$ and the polydispersity index $(\nexists)$ were calculated based on polyethylene glycol calibration standards. Nuclear Magnetic Resonance $\left({ }^{13} \mathrm{C}\right.$ - and ${ }^{1} \mathrm{H}$-NMR) was performed on a Varian $400 \mathrm{MHz}$ spectrometer. A refrigerated thermostatic bath (Alpha RA series) filled with water and ethylene glycol was operated at
$-10{ }^{\circ} \mathrm{C}$ or $-20{ }^{\circ} \mathrm{C}$ for gel freezing. Rheological studies were conducted on a controlled stress rheometer with $40 \mathrm{~mm}$ diameter parallel plate geometry (AR-G2, TA Instruments). For 3Dprinting, a 3D-Bioplotter (Developer Series, EnvisionTec, Germany) with Bioplotter RP software for file processing was used. Printing parameters: ambient temperature printing with $0.6 \mathrm{~mm}$ nozzle diameter, pressure of 4.0 bar, print head speed: $7.0 \mathrm{~mm} \mathrm{~s}^{-1}$, or high temperature printing $\left(80^{\circ} \mathrm{C}\right)$ with $0.4 \mathrm{~mm}$ nozzle diameter, pressure of 3.0 bar, print head speed: $12.0 \mathrm{~mm} \mathrm{~s}^{-1}$. Fourier transform infrared (FTIR) spectroscopy was performed on a Varian 7000 model, ATR mode. Confocal laser scanning microscopy (CLSM) was performed on a Nikon $\mathrm{A} 1 \mathrm{R}+$ microscope with a $40 \times$ objective using a laser excitation wavelength of $561 \mathrm{~nm}$. Data analysis was conducted with NIS elements viewer software. Thermogravimetric analysis (TGA) was conducted on a PerkinElmer Diamond instrument (Pyris Diamond TG-DTA).

\section{Synthesis of PHEAA-co-PMVK}

PHEAA-co-PMVK was synthesized by free radical polymerization. HEAA (3.0 g, $26.057 \mathrm{mmol})$, MVK (1.9 g, $27.464 \mathrm{mmol})$ and AIBN (0.007 g, $0.0426 \mathrm{mmol}$ ) were combined in a $100 \mathrm{~mL}$ round bottom flask and sealed with a rubber septum. The mixture was placed in an ice bath, degassed by nitrogen bubbling for $\sim 30 \mathrm{~min}$, sealed under nitrogen and placed in an oil bath heated to $60^{\circ} \mathrm{C}$. The mixture was left to react with stirring for $17 \mathrm{~h}$. The reaction was quenched by placing the flask in an ice bath. The solid-like contents of the flask were diluted with methanol and precipitated into diethyl ether. The product was collected, dried, and dissolved in deionized water. The aqueous solution was dialyzed, followed by freeze-drying, after which a solid product was obtained.

\section{Synthesis of tetraethylene glycol bishydroxylamine (TEG-BHA)}

The synthesis was performed according to a reported procedure. $^{26}$

\section{Preparation of 3D-printable self-healing oxime gels $\left(G_{\text {oxime }}\right)$}

PHEAA-co-PMVK (1.0 g, $10.44 \mathrm{mmol}$ ) was dissolved in $10.0 \mathrm{~mL}$ deionized water and allowed to stir at room temperature until a fully homogeneous solution was obtained. TEG-BHA $(6.0 \mathrm{mg}$, $0.02 \mathrm{mmol}$ ) was slowly introduced to the polymer solution under rigorous stirring and mixed for $10 \mathrm{~min}$. The gelation was indicated by a standard inversion test.

\section{Preparation of self-healing oxime gels with alternating degrees of cross-linking}

For the preparation of oxime cross-linked gels, PHEAA-coPMVK (0.1 g, $1.04 \mathrm{mmol}$ ) was dissolved in $1.0 \mathrm{~mL}$ deionized water and allowed to stir at room temperature until a fully homogeneous solution was obtained. TEG-BHA (0.9 mg, $0.003 \mathrm{mmol}$ ) was slowly introduced to the polymer solution under rigorous stirring and mixed for $10 \mathrm{~min}$. To obtain a lower degree of cross-linking, PHEAA-co-PMVK $(0.1 \mathrm{~g}$, $1.04 \mathrm{mmol}$ ) was dissolved in $0.9 \mathrm{~mL}$ deionized water and allowed to stir at room temperature until a fully homogeneous 
solution was obtained. TEG-BHA $(3.0 \mathrm{mg}, 0.01 \mathrm{mmol})$ was dissolved in $1.0 \mathrm{~mL}$ deionized water. Afterwards, $0.1 \mathrm{~mL}$ of TEG-BHA solution was introduced to the PHEAA-co-PMVK solution, stirred and allowed to set.

The gelation of the samples was indicated by a standard inversion test.

\section{Preparation of doubly dynamic self-healing cryogels}

$0.7 \mathrm{~mL}$ fractions of freshly prepared $G_{\text {oxime }}$ were loaded into a syringe and cast into glass vials to form uniform gel discs (diameter: $20.0 \mathrm{~mm}$, thickness: $5.0 \mathrm{~mm}$ ). The vials were sealed and allowed to stabilize overnight at ambient temperature. After overnight solidification, the gels were transferred to a cooling bath set to $-10^{\circ} \mathrm{C}$ or $-20^{\circ} \mathrm{C}$ for $24 \mathrm{~h}$. Afterwards, the gels were removed from the bath and allowed to thaw for $3 \mathrm{~h}$ at room temperature. Each freeze-thaw cycle was repeated as specified. These conditions were applied unless stated otherwise.

For the study of the effect of cryogelation time on the mechanical properties of the gels, each freezing cycle was conducted at $-10^{\circ} \mathrm{C}$ for $4 \mathrm{~h}$, followed by thawing at room temperature for $3 \mathrm{~h}$.

\section{Rheological characterization}

A time sweep was applied to measure the storage and loss modulus of the gels. The measurements were conducted at a constant temperature of $25^{\circ} \mathrm{C}$, an angular frequency of 6.283 rad $\mathrm{s}^{-1}$, and $0.5 \%$ strain. ${ }^{6}$ The measurements were repeated in triplicate. The degree of reinforcement $(R)$ was evaluated using formula (1):

$$
\% R=\frac{G_{n}^{\prime}-G_{0}^{\prime}}{G_{0}^{\prime}} \times 100
$$

where $G_{n}^{\prime}$ represents the average storage modulus of a gel after $n$ freeze-thaw cycles, and $G^{\prime}{ }_{0}$ represents the initial storage modulus of $G_{\text {oxime }}$ (without freeze-thaw treatment).

The self-healing characteristics of the gel were assessed using an increased strain ramp conducted at $25{ }^{\circ} \mathrm{C}$ and an angular frequency of $6.283 \mathrm{rad} \mathrm{s}^{-1}$. The flow properties of the gel (shear moduli as a function of stress, and apparent viscosity) were determined at 25 and $80{ }^{\circ} \mathrm{C}$ and an angular frequency of $6.283 \mathrm{rad} \mathrm{s}^{-1}$. A gelation kinetic study of $G_{\text {oxime }}$ was conducted by dissolving $0.1 \mathrm{~g}$ of PHEAA-co-PMVK in $1.0 \mathrm{~mL}$ deionized water. Afterwards, $0.6 \mathrm{mg}$ of TEG-BHA was added to the vial and stirred for a few minutes until fully homogenized. The contents of the vial were immediately transferred to the rheometer plate, followed by time sweep experiments conducted at $25{ }^{\circ} \mathrm{C}, 0.5 \%$ strain and an angular frequency of $6.283 \mathrm{rad} \mathrm{s}^{-1}$.

Frequency sweep was performed by examining the gel in the range of $0.01-10.0 \mathrm{~Hz}$ at $25^{\circ} \mathrm{C}$ and $1.0 \%$ strain.

\section{Mechanical testing $^{27}$}

Compression tests were performed on an Instron (Micro Tester $5848)$ equipped with a $5.0 \mathrm{~kg}$ load cell. Gel samples $(20.0 \mathrm{~mm}$ diameter, $5.0 \mathrm{~mm}$ thickness) were placed between the compression discs and deformed at a constant strain rate of $60 \%$ per minute. Young's (compression) modulus was extracted from stress-strain curves, by determining the slope of the curves in the low strain linear region (0-10\% strain). ${ }^{27}$ All the measurements were performed 3 times.

\section{Degree of swelling}

The gels were dried under vacuum for $24 \mathrm{~h}$, until no further change in their weight was observed. The weight of the samples was monitored. After drying, the gels were submerged in deionized water overnight, gently wiped to remove excess surface water and weighed. The degree of swelling $(S)$ was calculated according to formula (2): ${ }^{19,27}$

$$
\% S=\frac{W_{\mathrm{s}}}{W_{\mathrm{d}}} \times 100
$$

where $W_{\mathrm{d}}$ and $W_{\mathrm{s}}$ represent the weight of the dry and the swollen gels, respectively.

To examine the effect of $\mathrm{pH}$ on the degree of swelling, the same procedure was repeated with an acid (aqueous $\mathrm{HCl}$ solution, $\mathrm{pH}=4.5$ ) and a base (aqueous $\mathrm{KOH}$ solution, $\mathrm{pH}=8.5$ ) instead of deionized water.

\section{Porosity}

The evaluation of porosity $(P)$ was performed using the solvent replacement method. ${ }^{28}$ Briefly, the gels were dried under vacuum to constant weight. The dry weight was monitored. Afterwards, the dry gels were submerged in absolute ethanol overnight, wiped to remove excess solvent and weighed.

The porosity was calculated according to formula (3):

$$
\% P=\frac{W_{\mathrm{s}}-W_{\mathrm{d}}}{V \rho} \times 100
$$

where $W_{\mathrm{d}}$ and $W_{\mathrm{s}}$ are the weight of the dry and the swollen gel, respectively, $V$ is the volume of the gel, and $\rho$ is the density of absolute ethanol.

\section{Morphological characterization}

The morphology of the gels was characterized by CLSM to retain the hydrated state of the gels and to prevent pore collapse under vacuum. The gels were soaked in an aqueous solution of Rhodamine B (0.09 $\left.\mathrm{mg} \mathrm{mL}^{-1}\right)$ overnight. Afterwards, the gels were soaked in a deionized water reservoir and thoroughly washed several times to remove any excess dye. The pore size and wall thickness of the cryogels (average \pm stdev) were analysed using the NIS elements software.

\section{D-printing of $\boldsymbol{G}_{\text {oxime }}$}

$G_{\text {oxime }}$ was loaded into a disposable syringe equipped with a $0.6 \mathrm{~mm}$ nozzle and compressed until a good contact between the gel and the lid of the syringe was obtained and air evacuated. The syringe was placed into the 3D-Bioplotter machine, followed by standard purging and calibration procedures. Printing conditions (print head speed and pressure combination) were optimized to produce a stable, continuous and shape retaining filament strand (optimal printing conditions: 4.0 bar pressure, $7.0 \mathrm{~mm} \mathrm{~s}^{-1}$ print head speed, $25{ }^{\circ} \mathrm{C}$ ). In 
addition to ambient conditions, $G_{\text {oxime }}$ was also printed at $80{ }^{\circ} \mathrm{C}$ (stainless steel cartridge was used instead of a disposable plastic syringe). Optimal printing parameters: $0.4 \mathrm{~mm}$ nozzle diameter, 3.0 bar pressure, $12 \mathrm{~mm} \mathrm{~s}^{-1}$ printing speed.

\section{Post-printing (secondary) reinforcement by TIPS}

$G_{\text {oxime }}$ scaffolds $(100 \%$ infill, $2.0 \mathrm{~cm}$ diameter, $0.5 \mathrm{~cm}$ thickness) were $3 \mathrm{D}$-printed onto a glass slide and placed on the bottom of a glass vessel. After sealing, the vessel was placed in a thermoset controlled water bath set to $-10{ }^{\circ} \mathrm{C}$ or $-20{ }^{\circ} \mathrm{C}$ and subjected to a specified number of freeze-thaw cycles.

\section{Thermal stability analysis}

The thermal stability of PHEAA-co-PMVK was assessed by TGA. $6.9 \mathrm{mg}$ of PHEAA-co-PMVK were placed in an alumina crucible and the sample was heated at a constant rate of $10{ }^{\circ} \mathrm{C} \mathrm{min}^{-1}$ from $40{ }^{\circ} \mathrm{C}$ to $600{ }^{\circ} \mathrm{C}$ under a nitrogen flow of $200 \mathrm{~mL} \mathrm{~min}{ }^{-1}$.

\section{Results and discussion}

Engineering doubly dynamic, self-healing and 3D-printable gels requires a strategic design of a polymer system with mul- tiple functionalities and well-defined properties. A facile free radical copolymerization of cheap and accessible HEAA with MVK ( $\sim 40 \%$ yield, $M_{\mathrm{n}}=284 \mathrm{kDa}, D=2.39$ ) enabled the synthesis of a multi-functional platform in which the ketone groups of MVK act as reactive sites to form oxime cross-links (see Fig. S1-S3† for the ${ }^{1} \mathrm{H}-\mathrm{NMR}$ and ${ }^{13} \mathrm{C}-\mathrm{NMR}$ of PHEAA-coPMVK, Fig. S4 $\dagger$ for SEC traces of PHEAA-co-PMVK, and Fig. $\mathrm{S} 5 \dagger$ for ${ }^{1} \mathrm{H}-\mathrm{NMR}$ and a detailed synthesis procedure of TEG-BHA). Oxime chemistry has been chosen owing to its high hydrolytic stability ${ }^{29,30}$ and the ability to autonomously selfheal. ${ }^{3,31}$ The HEAA units of PHEAA-co-PMVK add a second dynamic functionality to the system by facilitating the formation of physical cross-links via hydrogen bonding during the cryogelation process. The excellent water solubility of HEAA eliminates the need for organic solvents and enables hydrogel formation in non-toxic aqueous media. The generalized method for the formation of 3D-printable, doubly dynamic, self-healing cryogels is presented in Scheme 1.

To be printable, gels must possess a good balance between the ability to flow and the ability to retain their 3D structure without collapsing. Therefore, a delicate rheological balance is required to formulate inks with optimal 3D-printing characteristics. ${ }^{6,12}$ Unlike commonly reported printing of non-covalently

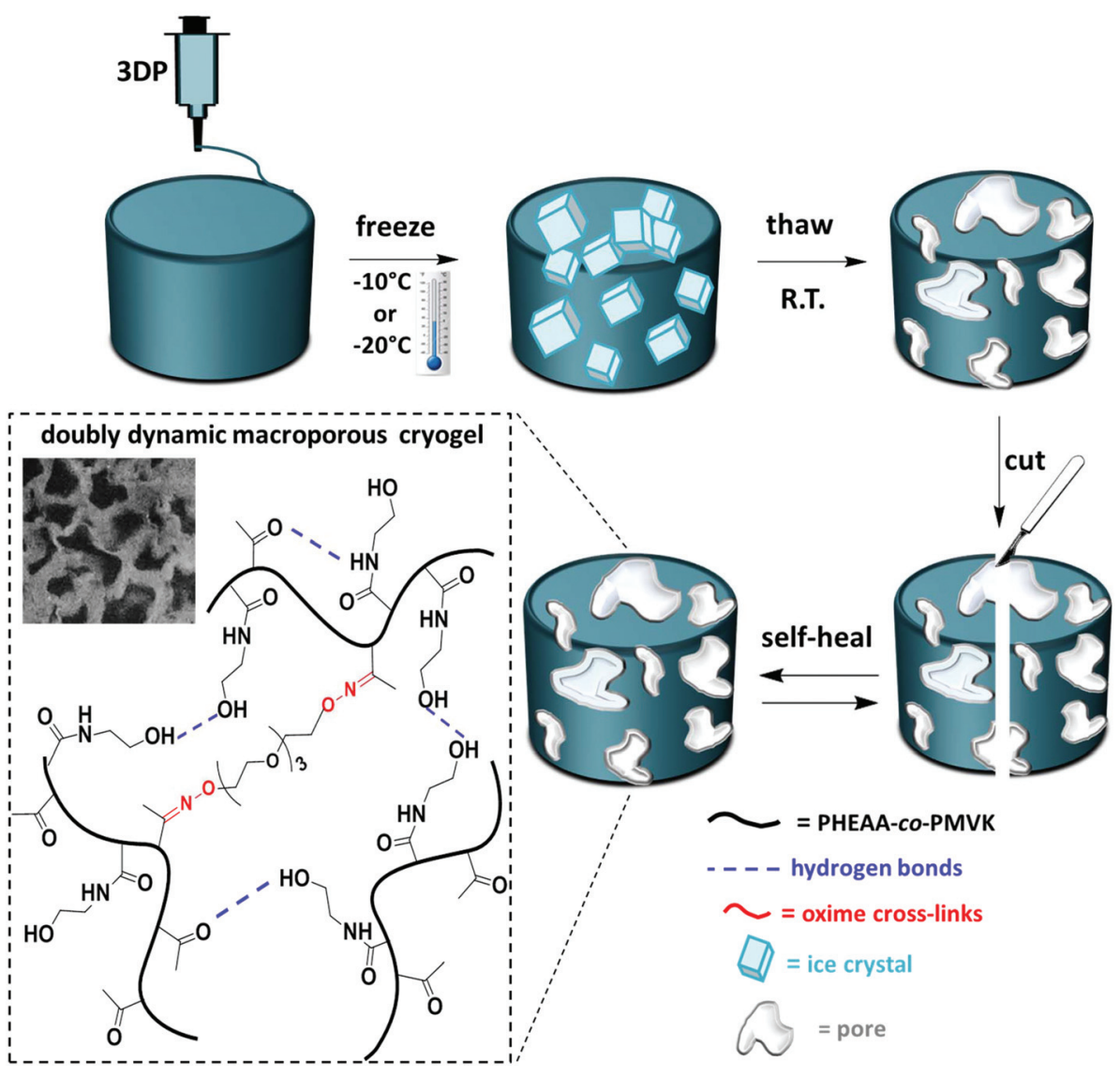

Scheme 1 A schematic of the methodology used to form 3D-printable, doubly dynamic self-healing cryogels. 3D-printing of oxime gels is followed by post-printing cryogelation, which induces the formation of macropores and physical cross-links by hydrogen bonding, and reinforces the object. The inset demonstrates the chemical structure of the cryogels and their macroporous morphology. 
cross-linked gels and polymer assemblies, ${ }^{11,13,21}$ 3DP of covalently cross-linked gels has the potential to be a challenging task due to a higher strength of covalent bonds, yet it remains desirable for the printing of materials requiring higher stability and diverse functionalities. The adjustment and optimization of oxime ink formulations was conducted according to an established approach. ${ }^{6} G_{\text {oxime }}$ was selected as an optimal formulation for $3 \mathrm{DP}$ in the current work. Rheological characterization of $G_{\text {oxime }}$ confirms its suitability for extrusion based 3DP (Fig. 1). The apparent viscosity measurements of $G_{\text {oxime }}$ indicate that the gel exhibits shear thinning behaviour, an essential criterion for extrudeability, and exhibits a good fit to a power law model ${ }^{32}$ (Fig. S6 $\dagger$ ). The increase in temperature from $25^{\circ} \mathrm{C}$ to $80^{\circ} \mathrm{C}$ resulted in a significant decrease in apparent viscosity, enabling the printing at more moderate pressures. Similar printing performances were obtained at $25{ }^{\circ} \mathrm{C}$ and $80{ }^{\circ} \mathrm{C}$. Printing at high temperatures was achievable due to the high thermal stability of PHEAA-co-PMVK, as indicated by the TGA (Fig. S7†).

Other important parameters for formulating extrudeable materials are the storage modulus and the point of flow associated with the yield stress. The applied shear stress generated in the print head must exceed the yielding point of the gel in order to induce flow. ${ }^{9}$ At ambient temperature, $G_{\text {oxime }}$ starts flowing at $\sim 1000 \mathrm{~Pa}$ and its storage modulus is $82 \mathrm{~Pa}$. At $80^{\circ} \mathrm{C}$, the stress required to induce $G_{\text {oxime }}$ flow decreases to $\sim 400 \mathrm{~Pa}$ (a)

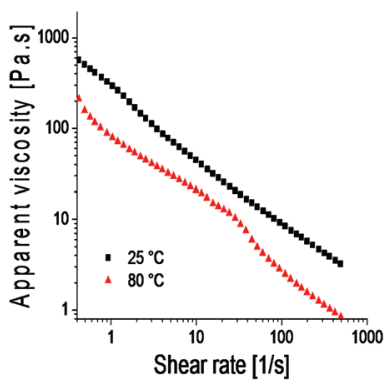

(c)

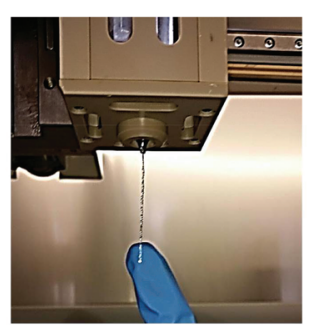

(b)

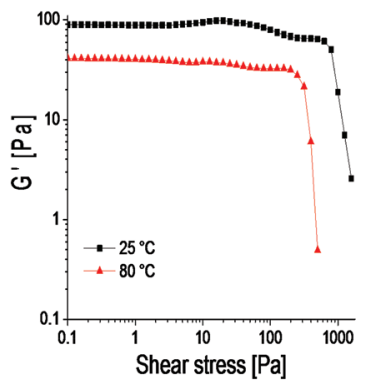

(d)

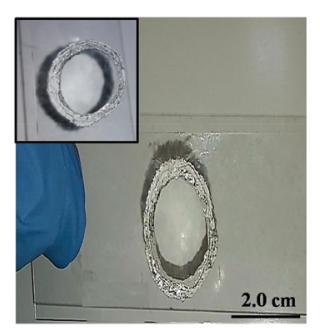

Fig. 1 Rheological characterization and the 3DP performance of $G_{\text {oxime: }}$ (a) apparent viscosity curves of $G_{\text {oxime }}$ at $25^{\circ} \mathrm{C}$ (black squares) and $80^{\circ} \mathrm{C}$ (red triangles). The gel exhibits shear thinning behaviour, suitable for 3DP. (b) Shear yielding of $G_{\text {oxime }}$ at $25^{\circ} \mathrm{C}$ (black squares) and $80{ }^{\circ} \mathrm{C}$ (red triangles). (c) A stable, shape-retaining extruded filament of $G_{\text {oxime. }}$ (d) 3D-printed hollow structure (6 layers of $G_{\text {oxime }}$ ink printed at $80^{\circ} \mathrm{C}$ on top of a glass substrate). The structure did not collapse upon substrate inversion, indicating its structural integrity. The inset demonstrates a caption taken from the top of the same hollow object. and the storage modulus to $40 \mathrm{~Pa}$ (Fig. 1b). The $G_{\text {oxime }}$ formulation is in an appropriate rheological range for successful 3Dprinting, and its rheological parameters are in agreement with previously reported values. ${ }^{6,9,11,12}$ The extrusion of $G_{\text {oxime }}$ resulted in the formation of well-structured and stable filamentous strands, suitable for layer-by-layer deposition (Fig. 1c). After the extrusion-induced shear was stopped, printed filaments exhibited fast shape recovery to well-defined self-supporting shapes. Although the 3DP of $G_{\text {oxime }}$ allowed fabricating only simple architectures, 3D-printed objects successfully retained their structure without collapsing, and enabled subsequent mounting of multiple ink layers on top of each other without spreading (Fig. 1d). Owing to the fast fusion of the filaments, solid and fully symmetrical infill patterns (100\% infill mesh), the printing directionality had no effect on the gels' properties.

Rapid, full and autonomous recovery from damage is a significant advantage for self-healing systems as they eliminate any need for the application of an external stimulus to fully heal the damage. The evaluation of $G_{\text {oxime }}$ and the cryogels' self-healing performance was conducted by using rheology measurements and induction of macroscopic cuts. Rheological analysis of $G_{\text {oxime }}$ shows that at high strains $(\sim 2200 \%)$ the network is destroyed. This trend is indicated by a sharp decrease in storage modulus, and the intersection of $G^{\prime}$ and $G^{\prime \prime}$. The self-healing (dynamic) response of the gel is indicated by the immediate recovery of $G^{\prime}$ to its initial values at low strain (Fig. 2a). Frequency sweep experiments revealed that $G_{\text {oxime }}$ exhibits frequency dependent behaviour, which is a characteristic of (albeit not unique to) self-healing gels (Fig. S8 $\dagger$ ). ${ }^{4,33}$ Finally, $G_{\text {oxime }}$ and the cryogel recovery from macroscopic cuts was examined. After the induction of a scalpel cut, the 2 halves of the gels were immediately brought into contact and the recovery of the gel to a single unit was followed (Fig. 2b-d and S9 $\dagger$ ). After $\sim 3$ hours at ambient temperature, and without any external additives, the damaged gels were healed and were able to support themselves under gravity. Owing to the dynamic nature of $G_{\text {oxime }}$ and its instant recovery, extrusion through a nozzle did not affect the gel. While oxime bonds can be disrupted by the printing shear, their ability to instantly recover from high mechanical strains enables them to retain their intact properties.

In addition to the $3 \mathrm{D}$-printable $G_{\text {oxime }}$, we have examined the effect of alternating degrees of cross-linking on the mechanical and self-healing properties of the gels (Fig. S10 $\dagger$ ). As expected, with the increase in the wt\% of TEG-BHA, stiffer gels were formed. The examined gels retained their selfhealing ability, and fully recovered from high-strain network destruction. However, these gels were not suitable for 3DP and were either too soft and spreadable, or densely cross-linked and therefore prevented a smooth mass flow during their extrusion.

The effect of post-printing treatment of soft $G_{\text {oxime }}$ by TIPS was explored and a structure-property investigation was conducted. Subjecting $G_{\text {oxime }}$ to subsequent freeze-thaw cycles was found to be an efficient method to increase the strength of 

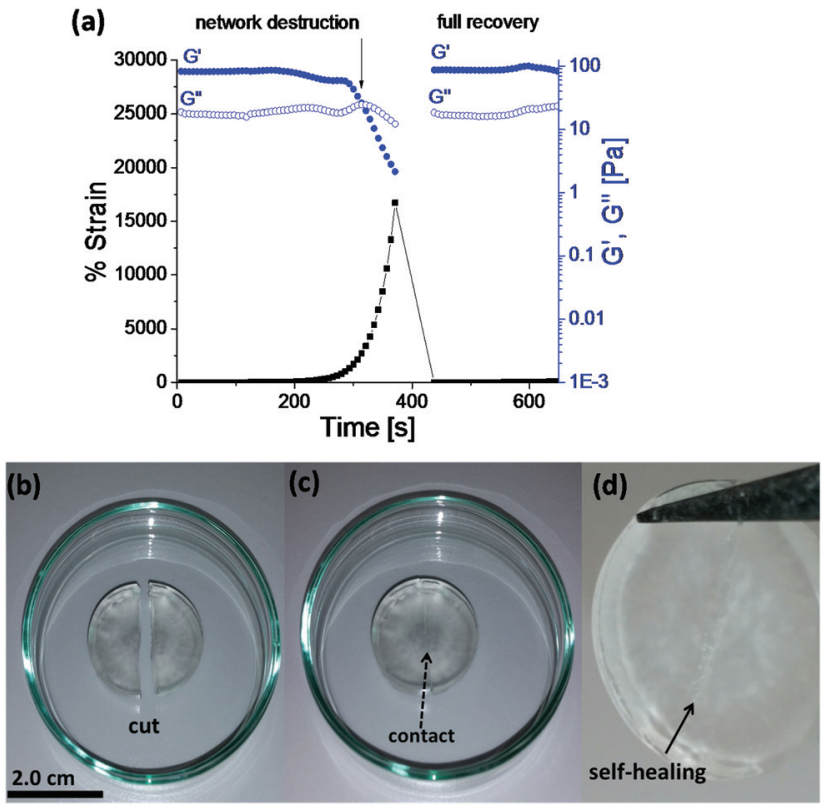

Fig. 2 Self-healing performances of $G_{\text {oxime }}$ and the doubly dynamic cryogels: (a) self-healing rheology of $G_{\text {oxime. }}$ The gel recovers from high strain destruction. The gel exhibits an immediate and full recovery. (b) 3D-printed $G_{\text {oxime }}$ scaffold after 3 cryogelation cycles at $-10{ }^{\circ} \mathrm{C}$ and induction of macroscopic cuts (a representative cryogel is demonstrated). (c) The damaged halves of the scaffold were brought into contact, to facilitate the healing process. (d) The damaged scaffold recovered from the cut, and could be lifted as a single self-supporting unit after $\sim 3 \mathrm{~h}$

$G_{\text {oxime }}$ in a well-controlled manner. The approach enabled a sensitive tuning of the mechanical properties through the precise control over TIPS conditions, such as freezing temperature, the number of cycles and the cycle's duration. The effect of TIPS treatment is demonstrated in Fig. 3. With the increasing number of cryogelation cycles a significant increase in storage modulus is observed, indicating the reinforcement effect of TIPS. Moreover, the reinforcement rate was highly dependent on the freezing temperature. When the freezing temperature was $-10{ }^{\circ} \mathrm{C}$, a higher reinforcement at a faster rate was achieved compared to freezing at $-20{ }^{\circ} \mathrm{C}$, (Fig. 3a and $\mathrm{b}$, respectively). Freezing at $-20{ }^{\circ} \mathrm{C}$ increased the storage modulus by up to $\sim 500 \%$ after 5 cycles. Freezing at $-10{ }^{\circ} \mathrm{C}$ resulted in a much more drastic storage modulus increase of up to $\sim 1900 \%$ after the same number of cycles. In both cases, the method allowed a very significant and gradual increase in the mechanical strength of $G_{\text {oxime}}$, demonstrating the advantages of this method in the reinforcement of soft materials. To investigate the effect of cryogelation time on the mechanical properties of $G_{\text {oxime }}$ the freezing time has been decreased from 24 to $4 \mathrm{~h}$. As expected, a longer cryogelation time resulted in a more significant reinforcement effect by enabling more time for the cross-links to form (Fig. S11†).

After TIPS treatment $\left(3\right.$ cycles or more at $-10^{\circ} \mathrm{C}$ or 5 cycles at $-20{ }^{\circ} \mathrm{C}$ ), the storage modulus of the cryogels becomes similar (or higher) to that of the previously reported non-
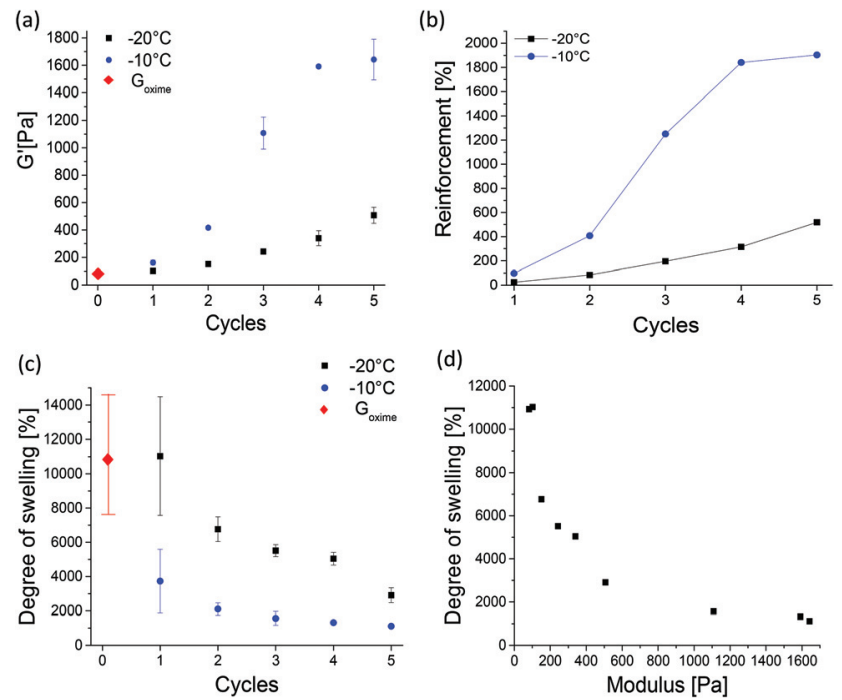

Fig. 3 Mechanical properties and swelling characteristics of $G_{\text {oxime }}$ and doubly dynamic, self-healing cryogels. (a) Storage modulus as a function of the number of freeze-thaw cycles at $-10{ }^{\circ} \mathrm{C}$ (blue circles) and $-20^{\circ} \mathrm{C}$ (black squares). The modulus increases with the number of cycles, indicating the reinforcement effect of the cryogelation process. (b) Reinforcement (\%) of cryogels prepared at $-10^{\circ} \mathrm{C}$ (blue circles) and $-20{ }^{\circ} \mathrm{C}$ (black squares). A significant increase in strength is observed with cryogelation cycles. (c) Degree of swelling of $G_{\text {oxime }}$ (red diamond), and the cryogels prepared at $-10{ }^{\circ} \mathrm{C}$ (blue circles) and $-20^{\circ} \mathrm{C}$ (black squares). A decrease in swelling with the number of cryogelation cycles was observed, indicating an increase in the degree of cross-linking. (d) A correlation between the mechanical strength and the degree of swelling of all the prepared cryogels demonstrates cryogelation-induced crosslinking.

printed self-healing gels. ${ }^{4,54}$ More importantly, unlike soft $G_{\text {oxime, }}$, the cryogelated objects could be easily handled, lifted and displaced from the substrate without spreading. After TIPS treatment the objects were reinforced, and therefore the cryogels are not printable. However, the cryogels remained complaint to some degree of deformation and shaping. The reinforcement effect of TIPS was also supported by compression tests, which are in good agreement with the rheological data, and indicate a gradual increase in Young's (compression) modulus with the increasing number of TIPS cycles (Fig. S12†). The reinforcement effect can be attributed to the formation of hydrogen bonding (physical) cross-links induced by the cryogelation process. It is well-known that cooling aqueous solutions containing cryogel precursors to subzero temperatures generates cryo-concentrated microphases, surrounded by ice crystals. ${ }^{35,36}$ These polymer rich zones facilitate interactions between polymer chains which can form physical cross-links. ${ }^{33,35}$ Since the polymer system contains multiple hydrogen bonding motifs, we hypothesized that the cryogelation process can facilitate physical cross-linking between the $G_{\text {oxime }}$ chains, and hence introduce a second dynamic functionality to the self-healing system. FTIR analysis of $G_{\text {oxime }}$ and the cryogels supports this hypothesis (Fig. S13 $\dagger$ ). The FTIR spectra indicate that after cryogelation, a significant broaden- 
ing and an increase in signal intensity of bound hydroxyl (2957-3687 $\mathrm{cm}^{-1}$ ) and carbonyl (1637 $\mathrm{cm}^{-1}$ ) groups occur. Moreover, the bound hydroxyl peak has shifted from 3367 to $3342 \mathrm{~cm}^{-1}$ as a result of cryogelation. These results provide evidence for hydrogen bonding enhancement by TIPS. In addition to the formation of physical cross-links by TIPS, it has recently been reported that low temperatures can actually increase the rate of oxime ligation. ${ }^{37}$ Agten et al. demonstrated that freezing can catalyse the formation of oxime ligation. ${ }^{37}$ Therefore, repeated freezing of $G_{\text {oxime }}$ may also account for the increased strength of $G_{\text {oxime }}$ after freezing cycles.

To investigate the stabilization of $G_{\text {oxime }}$, gelation kinetic studies were conducted. Rheological data indicate that the solto-gel transition occurs within $1 \mathrm{~h}$ (Fig. S14a $\dagger$ ). Re-examination of the same sample after overnight stabilization $(16 \mathrm{~h})$ revealed that $G^{\prime}$ values remained unchanged. This provides evidence of the full completion of the TEG-BHA cross-linking reaction under ambient conditions (Fig. S14b $\dagger$ ).

Unlike traditional types of cryogel precursors such as colloidal dispersions, monomeric, or polymeric precursors, ${ }^{35}$ the method described herein allows the formation of cryogels from gels and therefore represents a novel approach towards the formation of macroporous materials from solid, crosslinked precursors.

The changes in the degree of swelling of oxime cryogels provide additional evidence to confirm the formation of crosslinks by cryogelation. With an increasing number of freezethaw cycles, a decrease in swelling was observed (Fig. 3c). Moreover, an inverse correlation between the storage modulus and the degree of swelling of all the prepared cryogels was also observed (Fig. 3d). As expected, with the increasing number of subsequent cryogelation cycles, more cross-links are formed, which results in an increased mechanical strength and decreased swelling capabilities. The results are in good agreement with the literature. ${ }^{33} G_{\text {oxime }}$ and doubly dynamic selfhealing cryogels exhibit an extremely high degree of swelling. $G_{\text {oxime }}$ increased in weight by $\sim 11000 \%$ by water uptake, $G_{\text {oxime }}$ was subjected to 5 freeze-thaw cycles at $-10{ }^{\circ} \mathrm{C}$ and swelled by $\sim 1000 \%$, and $G_{\text {oxime }}$ was subjected to 5 freeze-thaw cycles at $-20{ }^{\circ} \mathrm{C}$ and swelled by $\sim 3000 \%$. The observed differences between the swelling capacities of the cryogels indicate that the cross-links remain stable and do not get disrupted by interactions with water. The swelling of $G_{\text {oxime }}$ and the cryogels in the acid and base was studied in order to assess their response to alternating $\mathrm{pH}$ values. When the gels were introduced to the acid $(\mathrm{pH}=4.5)$ and base $(\mathrm{pH}=8.5)$ their degrees of swelling decreased compared to the degrees of swelling in deionized water (Fig. S15a $\dagger$ ). In agreement with these observations, rheological examination of the gels confirmed the increase in their storage modulus in the acid and base (Fig. S15b $\dagger$ ). These results indicate that the acid and base can enhance the cross-linking of the gels and affect their interactions with water. The presence of an acid can catalyse the formation of oxime bonds ${ }^{38}$ by the protonation of ketone groups, making them more prone to nucleophilic attack by the unreacted TEG-BHA. Basic conditions had a similar effect on the gels. The understanding of the observed results under basic conditions requires a more detailed future investigation.

Rheological characterization of the cryogels indicates that the self-healing properties of the gels were not affected by TIPS, and the cryogels retained the ability to recover from network destruction (Fig. S16 $†$ ). Interestingly, the recovery tendency of $G_{\text {oxime }}$ and the cryogels were very similar and all the gels exhibited an immediate recovery. This similarity can be attributed to the methodological sensitivity, which does not provide a sufficient resolution to distinguish between these differences, especially when the recovery is instant and full. However, despite the similarity in the self-healing behaviour (recovery time and \%), the rheological response of the gels to the applied strain was different and strongly dependent on the mechanical strength of the gels. Soft gels $\left(G^{\prime} \sim 100 \mathrm{~Pa}\right)$ with a low degree of cross-linking ( $G_{\text {oxime }}$ and the cryogel prepared by 2 freeze-thaw cycles at $-20^{\circ} \mathrm{C}$, Fig. $2 \mathrm{a}$ and $\mathrm{S} 16 \mathrm{~b}, \dagger$ respectively) can withstand high strains $(\sim 2000 \%)$ before reaching a failure point. Highly cross-linked and stiffer cryogels $\left(G^{\prime} \sim 1000 \mathrm{~Pa}\right.$, cryogels prepared by 4 freeze-thaw cycles at $-10{ }^{\circ} \mathrm{C}$, Fig. S16a $\dagger$ ), on the other hand, fail at much lower strains ( $\sim 60 \%)$. Similar behaviour of soft and strong gels has been previously observed by Rutz et al. ${ }^{12}$ These differences can be explained by the fact that the polymer chains of softer gels (less cross-linked) are less constrained, and therefore have more freedom to deform and disentangle, requiring higher strains to reach their failure.

The differences between the degree of cross-linking and therefore the mechanical performance of oxime cryogels prepared at $-10{ }^{\circ} \mathrm{C}$ and $-20{ }^{\circ} \mathrm{C}$ can be explained by the cryogel microstructure. At lower freezing temperatures, the freezing rate is faster, and therefore the water crystalizes more rapidly,

Table 1 Summary of the mechanical, physical and morphological properties of $G_{\text {oxime }}$ and the doubly dynamic self-healing cryogels

\begin{tabular}{|c|c|c|c|c|c|c|c|c|}
\hline Entry & $\begin{array}{l}\text { Freezing } \\
\text { temperature }\left[{ }^{\circ} \mathrm{C}\right]\end{array}$ & $\begin{array}{l}\text { Number } \\
\text { of cycles }\end{array}$ & $\begin{array}{l}\text { Storage }^{a} \\
\text { modulus }[\mathrm{Pa}]\end{array}$ & $\begin{array}{l}\text { Young's }^{b} \\
\text { modulus }[\mathrm{Pa}]\end{array}$ & $\begin{array}{l}\text { Swelling }^{c} \\
\text { degree }[\%]\end{array}$ & $\begin{array}{l}\text { Porosity } \\
{[\%]}\end{array}$ & $\begin{array}{l}\text { Pore } \\
\text { size }[\mu \mathrm{m}]\end{array}$ & $\begin{array}{l}\text { Wall thickness } \\
{[\mu \mathrm{m}]}\end{array}$ \\
\hline$G_{\text {oxime }}$ & NA & NA & $82 \pm 2$ & $1243 \pm 831$ & $10925 \pm 3500$ & NA & NA & NA \\
\hline G1 & -10 & 2 & $416 \pm 9$ & $2730 \pm 1129$ & $2105 \pm 356$ & $64.0 \pm 3.6$ & $23.5 \pm 10.9$ & $7.4 \pm 1.8$ \\
\hline G2 & -20 & 2 & $151 \pm 5$ & $1700 \pm 306$ & $6665 \pm 722$ & $69.0 \pm 3.5$ & $6.4 \pm 4.5$ & $4.0 \pm 1.4$ \\
\hline G3 & -10 & 4 & $1591 \pm 13$ & $3213 \pm 402$ & $1319 \pm 44$ & $49.7 \pm 6.4$ & $16.7 \pm 3.5$ & $6.5 \pm 1.9$ \\
\hline G4 & -20 & 4 & $340 \pm 55$ & $2743 \pm 220$ & $5039 \pm 375$ & $45.0 \pm 4.2$ & $6.5 \pm 7.3$ & $2.9 \pm 1.0$ \\
\hline
\end{tabular}

${ }^{a}$ Determined by rheology tests (average $\pm \operatorname{stdev}, n=3$ ). ${ }^{b}$ Determined by compression tests (average \pm stdev, $n=3$ ). ${ }^{c}$ In deionized water. 

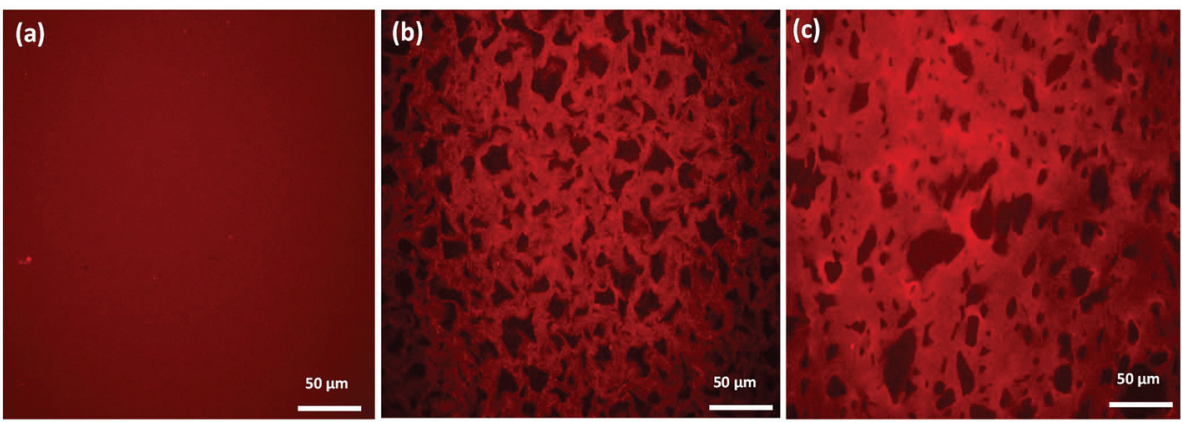

Fig. 4 CLSM morphological characterization of $G_{\text {oxime }}$ and the cryogels prepared at different freezing temperatures. (a) $G_{\text {oxime }}$ exhibits a nonporous structure. (b) $G_{\text {oxime }}$ subjected to cryogelation at $-10^{\circ} \mathrm{C}$, after 4 subsequent freeze-thaw cycles. The cryogel exhibits a macroporous structure with an average pore size of $16.7 \mu \mathrm{m}$ and an average wall thickness of $6.5 \mu \mathrm{m}$. (c) $G_{\text {oxime }}$ subjected to cryogelation at $-20^{\circ} \mathrm{C}$, after 4 subsequent freeze-thaw cycles. The cryogel exhibits a macroporous structure with an average pore size of $6.5 \mu \mathrm{m}$ and an average wall thickness of $2.9 \mu \mathrm{m}$. Lower cryogelation temperature results in weaker gels with a smaller pore size and wall thickness.

resulting in smaller crystals. Small water crystals are associated with a smaller pore size and thinner walls, as has been previously reported. ${ }^{35,36,39}$ CLSM indicates that the cryogels prepared at $-20{ }^{\circ} \mathrm{C}$ have a smaller pore size and thinner walls than the cryogels prepared at $-10{ }^{\circ} \mathrm{C}$ and are therefore mechanically weaker (Table 1). CLSM analysis of $G_{\text {oxime }}$ and the cryogels is presented in Fig. 4 and $\mathbf{S} 17 . \dagger$ While $G_{\text {oxime }}$ has a non-porous structure, the cryogels exhibit a sponge-like macroporous structure, which was generated by cryogelation. Ice crystals act as non-toxic and natural porogens, which leave well-defined pores after ice thawing. ${ }^{36}$ CLSM morphological analysis shows that a slower freezing rate also enables better control over the freezing process, which is reflected by a more uniform pore shape and size (Fig. 4b). In contrast, an extremely fast freezing rate results in more random pore morphology (Fig. 4c). All the parameters of $G_{\text {oxime }}$ and the doubly dynamic cryogels are summarized in Table 1.

\section{Conclusion}

We present doubly dynamic and self-healing cryogels with a macroporous structure and tuneable mechanical strength by TIPS. In conjunction with $3 \mathrm{DP}$, this method enables a facile post-printing reinforcement of soft 3D-printed gels, eliminating the need for additional chemicals or UV equipment. Soft 3D-printable gels are able to be reinforced by up to $\sim 1900 \%$, making soft 3D-printed objects strong and easily handled.

The mechanical strength of TIPS-treated gels can be modulated in a well-controlled manner via a specific choice of freeze-thaw conditions. Different freezing rates resulted in distinct microstructures of the cryogels and therefore different physical and mechanical characteristics. The formation of macroscopic interconnected pores was facilitated by hydrogen bonds between the PHEAA-co-PMVK chains, which could interact in polymer enriched macrophases during cryogelation. It was demonstrated that in addition to conventional cryogel precursors, solid gels can act as cryogel precursors. 3D-printable oxime gels and doubly dynamic cryogels prepared in this work exhibited rapid, full and autonomous recovery from damage (self-healing), making them promising functional materials for applications requiring extensive utilization and prolonged lifetimes. Moreover, their macroporous structure and tuneable mechanical properties make them promising candidates for biomedical applications. More importantly, we envision that the novel approach presented in this work is not limited to PHEAA-co-PMVK, and can be potentially implemented in other functional systems as a generalized reinforcement methodology.

\section{Funding sources}

This work has been supported by the Victorian Endowment for Science Knowledge and Innovation (LAC).

\section{Conflicts of interest}

The authors declare no conflict of interest.

\section{Acknowledgements}

The authors would like to thank Mr Paul Brannon from the Materials Characterization and Fabrication Platform for his assistance with CLSM, and Assoc. Prof. Andrea O'Connor for access to the Tissue Engineering Group lab facilities.

\section{References}

1 M. D. Hager, P. Greil, C. Leyens, S. Van Der Zwaag and U. S. Schubert, Adv. Mater., 2010, 22, 5424-5430.

2 Z. Wei, J. H. Yang, J. Zhou, F. Xu, M. Zrínyi, P. H. Dussault, Y. Osada and Y. M. Chen, Chem. Soc. Rev., 2014, 43, 81148131.

3 J. Collins, M. Nadgorny, Z. Xiao and L. A. Connal, Macromol. Rapid Commun., 2017, 38, 6. 
4 C. C. Deng, W. L. A. Brooks, K. A. Abboud and B. S. Sumerlin, ACS Macro Lett., 2015, 4, 220-224.

5 Y. Zhang, L. Tao, S. Li and Y. Wei, Biomacromolecules, 2011, 12, 1-7.

6 M. Nadgorny, Z. Xiao and L. A. Connal, Mol. Syst. Des. Eng., 2017, 2, 283-292.

7 J. R. Davidson, G. A. Appuhamillage, C. M. Thompson, W. Voit and R. A. Smaldone, ACS Appl. Mater. Interfaces, 2016, 8, 16961-16966.

8 D. M. Kirchmajer, R. Gorkin III and M. in het Panhuis, J. Mater. Chem. B, 2015, 3, 4105-4117.

9 R. L. Truby and J. A. Lewis, Nature, 2016, 540, 371-378.

10 W. Oropallo and L. A. Piegl, Eng. Comput., 2016, 32, 135148.

11 C. B. Highley, C. B. Rodell and J. A. Burdick, Adv. Mater., 2015, 27, 5075-5079.

12 A. L. Rutz, K. E. Hyland, A. E. Jakus, W. R. Burghardt and R. N. Shah, Adv. Mater., 2015, 27, 1607-1614.

13 L. R. Hart, S. Li, C. Sturgess, R. Wildman, J. R. Jones and W. Hayes, ACS Appl. Mater. Interfaces, 2016, 8, 3115-3122.

14 S. Hong, D. Sycks, H. F. ai Chan, S. Lin, G. P. Lopez, F. Guilak, K. W. Leong and X. Zhao, Adv. Mater., 2015, 27, 4034 .

15 M. Nadgorny, Z. Xiao, C. Chen and L. A. Connal, ACS Appl. Mater. Interfaces, 2016, 42, 28946-28954.

16 S. E. Bakarich, R. Gorkin and G. M. Spinks, Macromol. Rapid Commun., 2015, 36, 1211-1217.

17 G. I. Peterson, M. B. Larsen, M. A. Ganter, D. W. Storti and A. J. Boydston, ACS Appl. Mater. Interfaces, 2015, 7, 577-583.

18 S. Naficy, R. Gately, R. Gorkin, H. Xin and G. M. Spinks, Macromol. Mater. Eng., 2017, 302, 1-9.

19 S. E. Bakarich, M. in het Panhuis, S. Beirne, G. G. Wallace and G. M. Spinks, J. Mater. Chem. B, 2013, 1, 4939-4946.

20 R. R. Jose, J. E. Brown, K. E. Polido, F. G. Omenetto and D. L. Kaplan, ACS Biomater. Sci. Eng., 2015, 1, 780-788.

21 M. Zhang, A. Vora, W. Han, R. J. Wojtecki, H. Maune, A. B. A. Le, L. E. Thompson, G. M. McClelland, F. Ribet, A. C. Engler and A. Nelson, Macromolecules, 2015, 48, 64826488.
22 D. B. Kolesky, R. L. Truby, A. S. Gladman, T. A. Busbee, K. A. Homan and J. A. Lewis, Adv. Mater., 2014, 26, 31243130.

23 S. J. Shirbin, S. J. Lam, N. J. A. Chan, M. M. Ozmen, Q. Fu, N. O'Brien-Simpson, E. C. Reynolds and G. G. Qiao, ACS Macro Lett., 2016, 5, 552-557.

24 A. Di Luca, J. R. de Wijn, C. A. van Blitterswijka, S. Camarero-Espinosa and L. Moroni, Macromol. Rapid Commun., 2017, 38.

25 J. Collins, Z. Xiao, M. Müllner and L. A. Connal, Polym. Chem., 2016, 7, 3812-3826.

26 F. Taraballi, L. Russo, C. Battocchio, G. Polzonetti, F. Nicotra and L. Cipolla, Org. Biomol. Chem., 2014, 12, 4089-4092.

27 S. J. Shirbin, F. Karimi, N. J. A. Chan, D. E. Heath and G. G. Qiao, Biomacromolecules, 2016, 17, 2981-2991.

28 J. Y. Lai and Y. T. Li, Biomacromolecules, 2010, 11, 1387-1397. 29 R. T. Raines, Angew. Chem., Int. Ed., 2008, 47, 7523-7526.

30 J. Collins, Z. Xiao and L. A. Connal, J. Polym. Sci., Part A: Polym. Chem., 2017, 55, 3826-3831.

31 S. Mukherjee, M. R. Hill and B. S. Sumerlin, Soft Matter, 2015, 11, 6152-6161.

32 T. G. Mezger, The rheology handbook: for users of rotational and oscillatory rheometers, Vincentz Network GmbH \& Co KG, 2006.

33 H. Zhang, H. Xia and Y. Zhao, ACS Macro Lett., 2012, 1, 1233-1236.

34 Z. Wei, J. He, T. Liang, H. Oh, J. Athas, Z. Tong, C. Wang and Z. Nie, Polym. Chem., 2013, 4, 4601-4605.

35 O. Okay, Polymeric Cryogels, Springer, 2014.

36 T. M. A. Henderson, K. Ladewig, D. N. Haylock, K. M. McLean and A. J. O'Connor, J. Mater. Chem. B, 2013, 1, 2682-2695.

37 S. M. Agten, D. P. L. Suylen and T. M. Hackeng, Bioconjugate Chem., 2016, 27, 42-46.

38 A. Dirksen, T. M. Hackeng and P. E. Dawson, Angew. Chem., Int. Ed., 2006, 45, 7581-7584.

39 C. Oelschlaeger, F. Bossler and N. Willenbacher, Biomacromolecules, 2016, 17, 580-589. 